\title{
Erratum to: Is entheses ultrasound reliable? A reading Latin American exercise
}

\author{
L. Ventura-Ríos ${ }^{2}$ - V. Navarro-Compan ${ }^{1} \cdot$ M. Aliste $^{3} \cdot$ M. Alva Linares ${ }^{4} \cdot$ R. Areny ${ }^{5}$. \\ M. Audisio ${ }^{6}$ - A. M. Bertoli ${ }^{7}$ - T. Cazenave ${ }^{8}$ - C. Cerón ${ }^{9}$ - M. E. Díaz ${ }^{10}$ - M. Gutiérrez ${ }^{11}$. \\ C. Hernández ${ }^{2}$ - D. A. Navarta ${ }^{12}$. C. Pineda ${ }^{13}$ - G. E. Py ${ }^{6}$ - A. M. Reginato ${ }^{14}$ - J. Rosa ${ }^{8}$. \\ D. L. Saaibi ${ }^{15}$ - O. Sedano ${ }^{16}$ - C. Solano ${ }^{17}$ - C. Castillo-Gallego ${ }^{1}$. S. Falçao ${ }^{18}$. \\ E. De Miguel ${ }^{1}$
}

Published online: 28 August 2015

(C) International League of Associations for Rheumatology (ILAR) 2015

\section{Erratum to: Clin Rheumatol \\ DOI 10.1007/s10067-015-3007-x}

The original version of this article unfortunately contains an error in the name of one of the authors. The name L. V. Ríos should be presented as L. Ventura-Ríos. The correct author name is now presented in the authorgroup and affiliation sections.

The online version of the original article can be found at http://dx.doi.org/ 10.1007/s10067-015-3007-x.

E. De Miguel

eugenio.demiguel@gmail.com

1 Rheumatology Unit, La Paz University Hospital, $\mathrm{P}^{\circ}$ de la Castellana 261, 28046 Madrid, Spain

2 Musculoskeletal Ultrasound Laboratory, Instituto Nacional de Rehabilitación, México City, México

3 Rheumatology Unit, CentroMédico Clínica Santa María La Dehesa, Santiago, Chile

4 Rheumatology Unit, Hospital Edgardo Rebagliati Martins, Lima, Perú

5 Rheumatology Unit, Hospital Félix Bulnes Cerda-Universidad Mayor, Santiago, Chile

6 Rheumatology Unit, Facultad de Ciencias Médicas, Hospital Nacional de Clínicas, Córdoba, Argentina

7 Rheumatology Unit, Instituto Reumatológico Strusberg, Córdoba, Argentina

8 Rheumatology Unit, Instituto de Rehabilitación Psicofísica, Buenos Aires, Argentina

9 Reumatoligya SA, Rheumatology Medellin, Medellin, Colombia
10 Rheumatology Unit, Hospital Universitario Fundación Santa Fé de Bogotá, Bogotá, Colombia

11 Clinica Reumatologica, Universitá Politecnica delle Marche, Jesi, Ancona, Italy

12 Rheumatology Unit, Hospital Italiano de Buenos Aires, Buenos Aires, Argentina

13 Dirección de Investigación, Instituto Nacional de Rehabilitación, México City, México

14 Rheumatology Research and Musculoskeletal Ultrasound, Brown University, Providence VAMC, University Medicine Foundation/ RIH, Providence, RI, USA

15 Rheumatology Ubit, Centro Médico Carlos Ardila Lulle, Bucaramanga, Colombia

16 Rheumatology Lima, Hospital Marino Molina Scippa ESSALUD, Lima, Peru

17 Rheumatology Unit, Hospital Nacional Rosales, San Salvador, El Salvador

18 Serviço de Reumatologia do Centro Hospitalar de Lisboa Ocidental, EPE, Hospital Egas Moniz, Lisbon, Portugal 